\title{
Covert visual attention and extrafoveal information use during object identification
}

\author{
JOHN M. HENDERSON \\ University of Alberta, Edmonton, Alberta, Canada \\ and \\ ALEXANDER POLLATSEK and KEITH RAYNER \\ University of Massachusetts, Amherst, Massachusetts
}

\begin{abstract}
Three experiments are reported that examined the relationship between covert visual attention and a viewer's ability to use extrafoveal visual information during object identification. Subjects looked at arrays of four objects while their eye movements were recorded. Their task was to identify the objects in the array for an immediate probe memory test. During viewing, the number and location of objects visible during given fixations were manipulated. In Experiments 1 and 2, we found that multiple extrafoveal previews of an object did not afford any more benefit than a single extrafoveal preview, as assessed by means of time of fixation on the objects. In Experiment 3 , we found evidence for a model in which extrafoveal information acquired during a fixation derives primarily from the location toward which the eyes will move next. The results are discussed in terms of their implications for the relationship between covert visual attention and extrafoveal information use, and a sequential attention model is proposed.
\end{abstract}

During normal perception, the eyes tend to move in short hops or saccades, punctuated by fuxations during which the eyes remain relatively stationary and visual information acquisition takes place. This article is concerned with the acquisition and use of information from stimuli beyond the fovea (i.e., extrafoveal stimuli) during an eye fixation, given that the fixation occurs during a sequence of fixations and saccades (in contrast to tachistoscopic procedures). The acquisition of information from an extrafoveal stimulus can be seen as serving at least two distinct functions. First, extrafoveal processing is necessary for determining where to position future eye movements (see, e.g., Loftus, 1983; Morris, 1987; Rayner \& Pollatsek, 1987). Second, and more important for the purposes of this study, while an extrafoveal stimulus may not be fully analyzed before it is fixated, partial analysis of an extrafoveal stimulus often provides information that subsequently speeds the analysis of that stimulus once it

The research presented in this article was supported by NIMH Predoctoral Training Grant MH16745 to John M. Henderson, and by NSF Grant BNS86-09336 to Keith Rayner and Alexander Pollatsek. This research derives from a doctoral dissertation submitted by John $M$. Henderson to the University of Massachusetts. Aspects of the data were presented at the 1987 meeting of the Psychonomic Society in Seattle and the 1988 meeting of the Canadian Psychological Association in Montreal. The authors wish to thank Donald Fisher and Katherine Fite for serving on the dissertation committee, and Fernanda Ferreira, Raymond Klein, Lester Krueger, Geoffrey Loftus, Joan Gay Snodgrass, and several anonymous reviewers for commenting on earlier versions of the manuscript. Correspondence should be addressed to John $M$. Henderson, Department of Psychology, P-220 Biological Sciences Building, University of Alberta, Edmonton, Alberta, Canada T6G 2E9. is fixated (with respect to object identification, see Henderson, Pollatsek, \& Rayner, 1987; Pollatsek, Rayner, \& Collins, 1984; Pollatsek, Rayner, \& Henderson, 1988; with respect to word identification, see Balota, Pollatsek, \& Rayner, 1985; Lima, 1987; McConkie \& Zola, 1979).

The main issue in the present study is the relationship between covert visual attention and extrafoveal information acquisition, which we explored by examining the degree to which information useful to identification processes can be acquired from multiple extrafoveal locations during a fixation. Are all areas of the visual field beyond the fovea equally available for analysis by identification processes prior to a saccadic eye movement, or is information acquired only from some limited set of locations? There are several logical possibilities regarding the answer to this question. Two extreme views are: (1) that information is acquired from all extrafoveal locations (within the limits of acuity) during each fixation, and (2) that information is acquired from a single extrafoveal location during each fixation. The former possibility might be true if attention played little role and extrafoveal information acquisition were limited by acuity alone. On the other hand, an instantiation of the latter possibility is that information is available only from the location that is to be fixated next, for example if covert attention precedes the eye to the location about to be fixated (see Klein, 1980; McConkie, 1979; Morrison, 1984; Remington, 1980; Shepherd, Findlay, \& Hockey, 1986).

During normal scene viewing, objects occupy multiple extrafoveal locations, and an object may be seen several times extrafoveally before it is ultimately fixated. 
In Experiments 1 and 2, we addressed the issue of the availability of extrafoveal information by asking whether multiple extrafoveal glimpses afford more preview benefit than a single extrafoveal glimpse. In Experiment 3, we attempted to specify further the relationships among covert attention, eye movement direction, and the location from which extrafoveal information is acquired.

In order to examine the above issues, we used a variant of the moving window technique. This technique, originally developed by McConkie and Rayner (1975) in order to investigate similar issues in reading (see Rayner \& Bertera, 1979; Rayner, Inhoff, Morrison, Slowiaczek, \& Bertera, 1981; Rayner, Well, Pollatsek, \& Bertera, 1982), involves changing the display in real time contingent on the subject's point of fixation. In the present case, four objects were displayed at the corners of an imaginary square. The subject fixated each object in turn in order to identify it. In the various preview conditions employed, a subset of the four objects was displayed during a given fixation; in place of the other objects, a pattern mask appeared that served to mark their positions but provided no extrafoveal preview information about their identities.

The primary dependent measure was the mean duration of first fixation on an object (the duration of the first fixation on an object, excluding refixations within the object). In addition, the mean duration of gaze on an object (the overall time spent fixating an object before moving on to the next object, summed over successive fixations within an object) will also be reported. It has previously been argued that the mean first fixation duration primarily reflects object identification time (e.g., by Friedman, 1979; Henderson et al., 1987; Loftus \& Mackworth, 1978). It has also been proposed that the mean gaze duration primarily reflects postidentification processes such as memory integration (Inhoff, 1984). However, this strict dichotomy may be a bit too simplistic (Rayner \& Pollatsek, 1987). The decision to refixate an object in order to complete its identification must be made within the first fixation, yet the extra processing time a refixation allows will be reflected in the gaze duration, not the first fixation duration. This suggests that the safest position may be that first fixation durations may lead one to somewhat underestimate the effects of factors influencing identification processes, while gaze durations may similarly lead one to overestimate them. In keeping with this view, we will focus on the more conservative first fixation duration measure, but we will point out discrepancies between the two measures when they arise.

Aside from allowing us to evaluate the degree to which information can be acquired from multiple extrafoveal locations, the present paradigm also provides an opportunity to validate earlier estimates of the benefit provided by an extrafoveal preview during object identification. In earlier work, Pollatsek et al. (1984) and Henderson et al. (1987) attempted to provide a chronometric estimate of the benefit derived from an extrafoveal preview of an object, given that the object was subsequently fixated and identified. In both of these studies, the subjects fixated a central cross while a stimulus was presented extrafoveally. The subjects were instructed to move their eyes toward the extrafoveal stimulus as soon as it appeared, and to name it as quickly as possible. The recording of eye movements and the employment of a contingent display change technique enabled the original extrafoveal stimulus to be changed to another stimulus (a target object) during the saccade. An estimate of the amount of benefit derived from an extrafoveal preview of the target object was obtained through comparison of the naming latency when the extrafoveal stimulus was the same as the target object with the naming latency when the extrafoveal stimulus was different from the target object. Across a number of conditions, it was found that an extrafoveal preview at a distance of $5^{\circ}$ of visual angle from the center of fixation speeded naming latencies (and by inference, identification times) by between 100 and $140 \mathrm{msec}$, and at $10^{\circ}$, by between 40 and $130 \mathrm{msec}$.

The present paradigm has several advantages over the naming paradigm previously used by Pollatsek et al. (1984) and Henderson et al. (1987). First, since in the present study the subject was primarily concerned with identifying the object currently being fixated, it was possible to determine the extent to which information can be acquired from an extrafoveal object while processing resources are devoted to a foveal object. In the earlier studies, subjects were either not shown (Pollatsek et al., 1984) or were not required to identify (Henderson et al., 1987) a foveal object while they were acquiring extrafoveal information. To the extent that devoting processing resources to a foveal object reduces the amount of resources available for the processing of extrafoveal information, it may be that the necessity of identifying a foveal object would reduce the amount of benefit derived from an extrafoveal object. In addition, in the Henderson et al. and Pollatsek et al. studies there was no demand on processing resources beyond identification of the target object. In normal scene viewing, it may be that higher level processes (such as the construction of a scene level description or the encoding into memory of the recognized objects) require resources that decrease the usefulness of information from extrafoveal locations. Since the subjects in the current paradigm were asked to remember the four objects identified on each trial in order to answer a yes/no probe memory question at the end of the trial, it was possible to assess extrafoveal information use under more memory-intensive conditions.

A second methodological characteristic of the earlier studies also deserves consideration. Both earlier studies employed naming latency as an indication of identification time. In the current paradigm, no overt response was required of the subject. Instead, the dependent measure was the time of fixation on each object. The fixation time measure has several advantages over the naming response. First, it does not require the generation of a name code-a stage of processing that may not be involved in normal object identification (Huttenlocher \& Kubicek, 1983; Kroll \& Potter, 1984). Second, it is a noninvasive mea- 
sure, allowing subjects to view the display in a natural manner. Third, fixation times tend to be shorter than naming latencies for objects, and for this reason they may constitute more of an "on-line" measure of object recognition and be less susceptible to postidentification influences.

\section{EXPERIMENT 1}

Four objects were presented on each trial in this experiment. Therefore, the present paradigm allows for the examination of the possible cumulative effects of extrafoveal information across several extrafoveal previews. Two general classes of theories concerning multiple extrafoveal glimpses can be considered. According to one class, extrafoveal information is cumulative, so several extrafoveal previews provide more information about an object than a single preview. We will call this the cumulative preview hypothesis. On the other hand, it could be that a single extrafoveal preview provides as much information about an object as can be acquired short of an actual fixation on the object. In this case, subsequent extrafoveal glimpses can provide no further information. We will call this the limited preview hypothesis.

In this experiment, subjects were shown displays of four objects arranged in an imaginary square and were asked to look at the displays while their eye movements were recorded. The subjects were instructed to look at the four objects in each display in a prescribed order, and then to press a key to terminate the display. The subjects were told that they should look at each display until they knew what the four objects were. After terminating the display, each subject was verbally given the name of an object by the experimenter and asked to indicate whether that object had appeared in the display just examined.

The primary manipulation in this experiment was the presence or absence of extrafoveal preview information. In the full display condition, all four objects were continually visible until display termination. In the one-object condition, only the fixated object was visible, while each of the other three objects was replaced by a pattern mask in the same position and of roughly the same size as the object. An estimate of the extrafoveal preview benefit, or savings in object identification time due to having had an extrafoveal preview of an object, could be derived by comparing mean durations of first fixation on objects that had been shown extrafoveally with those that had not. Since this paradigm requires that a foveal object be recognized and remembered at the same time as extrafoveal information is acquired, it allows for a converging estimate (along with the results of Henderson et al., 1987, and Pollatsek et al., 1984) of the extrafoveal preview benefit under more natural conditions.

Because four objects were included in each display, it was possible to examine any cumulative effects of several extrafoveal previews of an object. If preview information can be acquired from all extrafoveal locations during a given fixation, then objects fixated later in the display should show a greater extrafoveal preview benefit than objects fixated earlier. Since it was necessary for subjects to fixate all four objects on most trials, it was possible to examine cases in which there were from one to four extrafoveal previews of an object prior to fixation.

\section{Method}

Subjects. Six members of the University of Massachusetts subject pool participated in Experiment 1 . All of the subjects had previously participated in eye movement experiments and were therefore comfortable with the eye tracking procedure.

Materials. The stimuli were 24 line drawings of common objects, mostly taken from Snodgrass and Vanderwart (1980). Before the experiment, the subjects were presented each object one at a time, and they were asked to name them. If necessary, the $\mathrm{cx}$ perimenter corrected the name employed; this prevented confusion during the experiment. The 24 objects were combined into 12 displays, each containing 4 semantically unrelated objects. The objects in each display were centered on the corners of an imaginary square. Each object subtended approximately $2^{\circ}$ of visual angle both horizontally and vertically, and there were about $4.5^{\circ}$ of visual angle between the centers of any two adjacent objects (see Figure 1). In addition, a pattern mask composed of irregularly drawn line segments was employed in the one-object condition. This mask was slightly larger than the largest object. All drawings were entered into the computer via a Summagraphics Bit-Pad.

Apparatus. The stimuli were displayed on a Hewlett-Packard 1300 A cathode-ray tube (CRT) with a P-31 phosphor. Removing a point on the CRT results in a drop to $1 \%$ of maximum brightness in $0.25 \mathrm{msec}$. A black theater gel covered the CRT so that the display appeared clear and sharp to the subjects. A bite bar was used to eliminate head movements.

Eye movements were monitored via a Stanford Research Institute Dual Purkinje Eyetracker, which has a resolution of about 10' of arc. The eyetracker and CRT were interfaced with a HewlettPackard 2100 computer, which controlled the experiment. During the experiment, the computer kept a complete record of the subject's eye movement behavior, including fixation position and fixation duration. The signal from the eyetracker was sampled every $1 \mathrm{msec}$ by the computer, and the position of the eye was determined
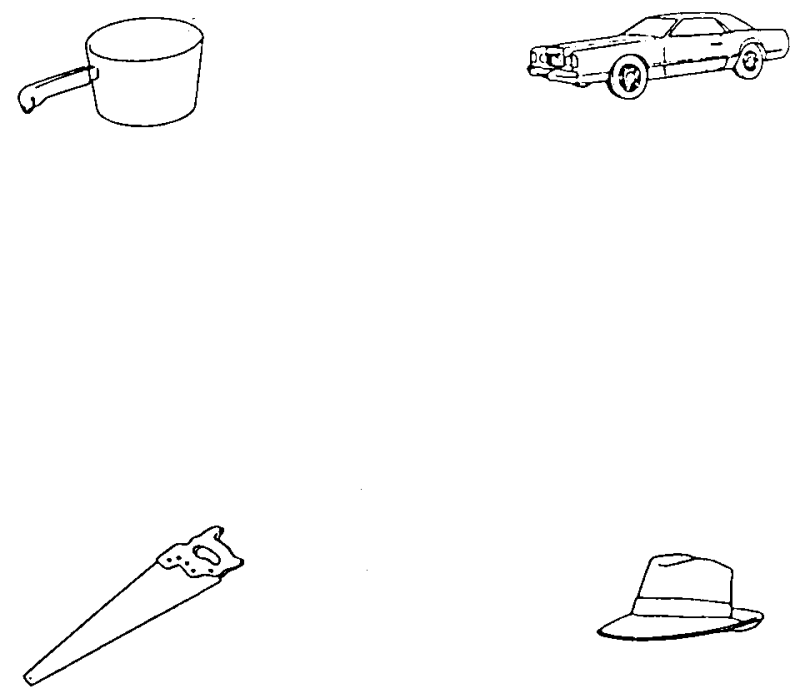

Figure 1. Example of the layout of the stimulus displays used in Experiments 1 through 3. Each object subtended about $2^{\circ}$ of visual angle vertically and horizontally, with about $4.5^{\circ}$ between the centers of adjacent objects. 
every $4 \mathrm{msec}$. The program controlling the experiment divided the display into five areas, four of these areas equivalent to the four quadrants in which an object could occur, and one area at the center of the display. In the one-object condition, a pattern mask occurred at each of the four object positions until the subject moved his/her eyes from the center. At that time, the object occupying the quadrant in which the eyes were moving was displayed. Thereafter, as soon as the eyes crossed a quadrant boundary, the object in that quadrant was displayed, and all other objects were replaced by the masks. Since the display change required no longer than $5 \mathrm{msec}$ once the subject's eyes crossed the quadrant boundary, it was almost always completed during the saccade and so was not seen by the subject.

The subject's eyes were $46 \mathrm{~cm}$ from the CRT. Eye movements were monitored from the right eye, but viewing was binocular. The room was dark except for the displays themselves and a dim indirect light source.

Procedure. Upon arriving for a session, each subject was seated comfortably before the CRT. The eye movement system was then calibrated in both the horizontal and vertical dimensions. After calibration, two blocks of 12 test trials were given. ${ }^{1}$ Each trial consisted of the following events: first, a central fixation cross appeared, and the calibration was checked by examining the position of a second cross that moved with the eye. If the calibration was satisfactory, the experimenter warned the subject that the trial was about to begin, and approximately $250 \mathrm{msec}$ later the fixation and calibration crosses were replaced by a display. The subject then made a saccade from the center of the display (where there was no object) to the upper left position, and thereafter looked around the display in a counterclockwise direction in order to see which objects were there. All subjects found the prescribed order in which to view the four objects easy and natural to follow. ${ }^{2}$ The subjects were told that they could look back to an object if necessary, but they were encouraged to maintain the prescribed viewing order if possible. When a "regression" (an eye movement to a previously viewed object) occurred in the one-object condition, the program effected the appropriate display change.

When the subject felt that he or she had identified the objects in the display, he or she pushed a display termination key. This caused the objects to disappear and be replaced by four pattern masks for $500 \mathrm{msec}$, one in each object position. These masks were used in order to make it impossible to answer the probe question on the basis of phosphor persistence. The experimenter then asked the subject whether a particular object had appeared in that display. Half of the questions required a "yes" response, and half a "no" response. The subject was queried about each of the four object positions equally often. These questions were included in order to ensure that the subject was encoding the objects; the subjects had no difficulty in answering the questions correctly. ${ }^{3}$

The primary dependent measure was the duration of first fixation on the objects at each ordinal object location. While the data from all of these possible ordinal object positions were examined, an a priori decision was made to focus on the second and third objects fixated in each display. This decision was made because the first object was fixated following a close preview in the center of the display where there was no foveal object, and the fixation on the last object was terminated by a manual response to the display termination key rather than by an eye movement.

Each subject participated in two blocks of trials. In the first block, the subject saw all test displays in a random order. After a short rest, the subject received the second block, which consisted of the same displays in a new random order. For 3 of the subjects, the first block consisted of the full display condition and the second block consisted of the one-object condition. For the other 3 subjects, the order of conditions was reversed. The entire experiment lasted from 30 to $45 \mathrm{~min}$.

\section{Results and Discussion}

The following analyses exclude trials on which (1) the eyetracker lost track of the eye (3\%), and (2) an object was skipped (1\%) or fixated for less than 50 msec or more than 1200 msec $(1 \%)$.

As can be seen in Table 1, the mean duration of first fixation on the four objects in the one-object condition was $354 \mathrm{msec}$, while the mean duration of first fixation on these objects in the full display condition was $255 \mathrm{msec}$. Therefore, when the subjects were given an extrafoveal preview of the objects, first fixation durations were 99 msec shorter than when no preview was given $[F(1,5)=27.74, p<.005]$. The magnitude of this preview effect is similar to those of the preview effects observed in earlier studies using naming latency as a measure of identification time. These data therefore provide converging evidence for the view that a great deal of useful visual information about an object can be acquired from beyond the fovea and subsequently integrated with information acquired once the object is fixated. In the Pollatsek et al. (1984) experiments, the average preview benefit at a $5^{\circ}$ eccentricity across six experiments was $128 \mathrm{msec}$. In the two experiments in which Henderson et al. (1987) examined extrafoveal preview benefit, the preview effects at $5^{\circ}$ were 103 and $106 \mathrm{msec}$. In neither of these earlier studies were the subjects required to identify a foveal object at the same time as they were acquiring extrafoveal information. The 99-msec preview effect found in the present experiment suggests that a substantial amount of information about objects can be acquired from extrafoveal locations even when a foveal object must be identified and remembered.

The primary purpose of Experiment 1 was to determine whether extrafoveal preview benefit would be cumulative across multiple previews, or whether it would tend to be limited to a particular level regardless of the number of previews. There would seem to be two ways of examining this issue from the present data. First, all things being equal, if the cumulative hypothesis is correct, the objects in the full display condition should be identified faster as ordinal position increases. This prediction follows because an object in the first position will only have been seen extrafoveally once, while an object in the second position will have been seen extrafoveally twice, and so on. As can be seen in Table 1, there was no obvious tendency

Table 1

Mean First Fixation Duration (in msec) by Preview Condition and Ordinal Object Position, Experiment 1

\begin{tabular}{lccccc}
\hline & \multicolumn{4}{c}{ Ordinal Object Position } \\
\cline { 2 - 5 } Preview Condition & 1 & 2 & 3 & 4 & Mean \\
\hline One-object & 410 & 386 & 293 & 326 & 354 \\
& $(434)$ & $(401)$ & $(366)$ & $(343)$ & $(386)$ \\
Full display & 246 & 273 & 251 & 251 & 255 \\
& $(275)$ & $(279)$ & $(261)$ & $(256)$ & $(268)$ \\
Preview benefit & 164 & 113 & 42 & 75 & 99 \\
& $(159)$ & $(122)$ & $(102)$ & $(87)$ & $(118)$ \\
\hline
\end{tabular}

Note-Mean gaze durations are given in parentheses. 
for this to occur, and an analysis of variance conducted on the full display condition alone showed no ordinal position effect $(F<1)$.

It is possible that in the analysis above, a cumulative preview effect might be underestimated. For example, suppose that the amount of extrafoveal information does increase across multiple previews, but that the savings in identification time afforded by this increase is offset by the slower processing of the object once it is fixated. Such slower processing might be due to the higher memory load caused by the requirement of remembering each additional object as it is identified. If this is the case, then the difference in first fixation durations in the one-object and full display conditions across ordinal object position provides a more direct test, since the memory load is constant across the one-object and full display conditions. The cumulative preview hypothesis predicts that the difference in the one-object and full display conditions should increase across ordinal position. While there was no main effect of ordinal object position $[F(3,15)=1.37$, $p>.25$ ], ordinal object position did influence the amount of preview benefit derived $[F(3,15)=3.94, p<.05]$. However, as can be seen in Table 1 , aside from the increase in preview benefit from the third to the fourth ordinal positions, the trend was in the direction opposite to that predicted by the cumulative preview hypothesis.

Because preview information about the first object in each display was acquired while the subject fixated the center of the display (which was closer to the previewed object and included no foveal object), and because the duration of fixation on the last object in each display may have been contaminated by the display termination key response, the two intermediate ordinal positions provide the cleanest comparison for examining possible cumulative effects of preview. The main analysis therefore compared just these two ordinal object positions. There was an average $78-\mathrm{msec}$ preview effect at these positions $[F(1,5)=9.28, p<.05]$, and the mean first fixation duration decreased from $330 \mathrm{msec}$ at Ordinal Position 2 to $272 \mathrm{msec}$ at Ordinal Position $3[F(1,5)=7.57, p<.05]$. Most importantly, however, the preview benefit was larger in the second ordinal position than in the third position $[F(1,5)=6.94, p<.05]$, contrary to the prediction of the cumulative preview hypothesis.

The present experiment does not support the hypothesis that extrafoveal preview information accumulates over multiple previews. As can be seen in Table 1, both the mean first fixation duration data and the mean gaze duration data (given in parentheses) converge on this conclusion. The general trend instead appears to be a reduction in preview information across multiple extrafoveal glimpses of an object, and the cleanest comparison, between Ordinal Positions 2 and 3, supports this conclusion. On the basis of these results, the cumulative preview hypothesis appears untenable.

Before accepting the results of Experiment 1 as definitive, we must consider a possible artifactual explanation for the lack of a cumulative preview benefit. Because of an attempt to keep the number of repetitions of a given object to a reasonable minimum, each object appeared in only one object position across displays. Therefore, object and ordinal object position were confounded. Although it seems unlikely, it is possible that the objects chosen at each increasing ordinal object position were inherently less able to provide preview benefit, thus offsetting a true cumulative preview benefit. Experiment 2 was conducted in order to rule out this possibility.

\section{EXPERIMENT 2}

The main purpose of Experiment 2 was to replicate Experiment 1 with a new random assignment of objects to ordinal object position that would reduce the likelihood of an artifactual explanation for the lack of a cumulative preview benefit. Experiment 2 also served to replicate Experiment 1 with a larger set of objects and test displays, so that we might determine whether the decrease in extrafoveal preview benefit across ordinal object positions was reliable.

\section{Method}

Subjects. Eight members of the University of Massachusetts subject pool participated in Experiment 2. All subjects had previously been in eye movement experiments, and 2 of the 8 subjects had participated in Experiment 1.

Materials. The stimuli were 72 line drawings of common objects taken from the same pool as were those used in Experiment 1. The subjects were again asked to name the individually presented objects prior to the experiment. The 72 objects were combined into 54 displays of 4 semantically unrelated objects each. Of these 54 displays, 30 were considered test trials for the purposes of this experiment (the other trials were part of a separate study and will not be discussed here). Across the 54 displays, each of the 72 objects occurred an equal number of times. As in Experiment 1, each particular object did not appear at all ordinal object positions. However, objects that had appeared in Experiment I were randomly assigned to a new position in Experiment 2. This, in conjunction with the fact that a larger group of objects was employed overall, ought to have helped mitigate against any effect due to the particular objects used at each ordinal position.

Apparatus and Procedure. The apparatus and procedure were identical to those used in Experiment 1. The entire experiment lasted from 45 to $60 \mathrm{~min}$.

\section{Results and Discussion}

The following analyses exclude trials on which (1) the eyetracker lost track of the eye (1\%), and (2) an object was skipped (1\%) or fixated for less than $50 \mathrm{msec}$ or more than $1200 \mathrm{msec}(2 \%)$.

The mean duration of first fixation on the four objects of each display in the one-object condition was $430 \mathrm{msec}$, while the mean duration of first fixation on these objects in the full display condition was $340 \mathrm{msec}$. Therefore, first fixation durations were $90 \mathrm{msec}$ shorter when the objects were available extrafoveally before fixation than when no preview was given $[F(1,7)=28.88, p<.005]$. The magnitude of this preview effect is very similar to that of the preview effect observed in Experiment 1 and to those in the earlier studies. The fact that overall fixa- 
tion times were longer in Experiment 2 than in Experiment 1 was probably due to the fact that a larger set of objects was used in Experiment 2, as well as to the particular objects chosen.

As can be seen in the second row of Table 2, there was again no tendency for the mean duration of first fixation on objects to decrease when an extrafoveal preview was available across ordinal object position $[F(3,21)=1.68$, $p>.20$ ], counter to the prediction of the cumulative preview hypothesis. In addition, comparison of the crucial second and third ordinal object positions supports this conclusion, since there was only a nonsignificant 6-msec difference between the first fixation durations at these two ordinal positions when a preview was available $(F<1)$. The gaze duration data generally followed the same pattern as the first fixation duration data except for the slight increase in the mean gaze duration from the second to the third ordinal object positions. This difference, however, did not approach significance $(F<1)$.

The pattern of the preview effect (the difference between the one-object and full display conditions) reflected in first fixation durations across ordinal object position generally replicated the pattern observed in Experiment 1 . There was an overall effect of ordinal object position $[F(3,21)=3.22, p<.05]$, primarily due to the increase in fixation durations at the fourth ordinal position, and there was a marginal interaction between the preview and ordinal position conditions $[F(3,21)=2.86, p=.06]$. As Table 2 shows, this marginal interaction did not support the cumulative preview hypothesis, since there was again no obvious tendency for extrafoveal preview benefit to increase, except perhaps at the contaminated fourth ordinal position. Looking at just the second and third ordinal positions, the overall 66-msec preview effect was significant $[F(1,7)=6.53, p<.05]$. The 31 -msec decrease in preview benefit from the second to the third ordinal positions was not significant $[F(1,7)=1.28, p>.25]$, and in any case it was in the direction opposite to that predicted by the cumulative preview hypothesis, replicating the general pattern found in Experiment 1. Again, the gaze duration data generally followed the same pattern as the first fixation duration data, apart from the nonsignificant increase in preview benefit from the second to third ordinal object positions $(F<1)$. Thus the data again suggest that the amount of preview benefit gained from

Table 2

Mean First Fixation Duration (in msec) by Preview Condition and Ordinal Object Position, Experiment 2

\begin{tabular}{lccccc}
\hline & \multicolumn{4}{c}{ Ordinal Object Position } & \\
\cline { 2 - 5 } Preview Condition & 1 & 2 & 3 & 4 & Mean \\
\hline One-object & 372 & 437 & 400 & 511 & 430 \\
& $(744)$ & $(580)$ & $(654)$ & $(654)$ & $(658)$ \\
Full display & 297 & 355 & 349 & 357 & 340 \\
& $(529)$ & $(437)$ & $(471)$ & $(478)$ & $(479)$ \\
Preview benefit & 75 & 82 & 51 & 154 & 90 \\
& $(215)$ & $(143)$ & $(183)$ & $(176)$ & $(179)$ \\
\hline
\end{tabular}

Note-Mean gaze durations are given in parentheses. an extrafoveal object does not increase with multiple extrafoveal glimpses.

\section{Discussion of Experiments 1 and 2}

Several general conclusions regarding the use of extrafoveal information during object identification can be made on the basis of Experiments 1 and 2. First, the chronometric estimates of the benefit derived from an extrafoveal preview of an object in these experiments generally corroborated the estimates derived from previous studies (Henderson et al., 1987; Pollatsek et al., 1984). In the earlier studies, naming latencies were used as an indication of the time needed to identify an object, and it was found that an extrafoveal preview of an object at a $5^{\circ}$ eccentricity afforded subjects a savings of 100 to $140 \mathrm{msec}$ in identification time. In the present experiments, where mean first fixation duration was used as an identification time estimate, the overall savings derived from a $5^{\circ}$ preview was $99 \mathrm{msec}$ (Experiment 1) and $90 \mathrm{msec}$ (Experiment 2). These estimates of the preview benefit derived from the first fixation duration data were generally more conservative than the estimates derived from the gaze duration data. The gaze duration estimates of the preview benefit were $118 \mathrm{msec}$ and $179 \mathrm{msec}$ in Experiments 1 and 2, respectively.

Continuing with the more conservative estimate of the savings due to an extrafoveal preview, an examination of the preview benefit averaged over the second and third ordinal positions alone shows a preview benefit of 78 and 66 msec in Experiments 1 and 2, respectively, using first fixation durations. Given that these ordinal positions probably provide the cleanest estimates of the preview benefit, it could be argued that they most accurately reflect the amount of benefit that can be derived from an extrafoveal preview when subjects are required to identify and remember an object in the fovea at the same time as they are acquiring extrafoveal information. Since in the Henderson et al. (1987) and Pollatsek et al. (1984) studies subjects were not required to identify a foveal object, the cross study comparison (which of course must be viewed with caution) suggests that there may be a decrement in the ability to use preview information when some attentional resources are involved with identifying and remembering a foveal object at the same time as extrafoveal information is being acquired. This possibility is further supported by experiments conducted using both line drawings (Henderson, 1988) and words (Henderson \& Ferreira, 1987), in which foveal processing difficulty was directly manipulated, and a reduction in the acquisition of extrafoveal information was found (see also Rayner, 1986).

The main issue explored in Experiments 1 and 2 was the manner in which the visual system processes multiple extrafoveal previews of an object. Two general hypotheses were originally considered. According to the cumulative preview hypothesis, added benefit ought to have been derived from each additional extrafoveal preview, whereas the limited preview hypothesis posited 
that a single preview should provide as much information as could be acquired prior to fixation. The data from both Experiments 1 and 2 were found to be inconsistent with the cumulative preview hypothesis. Averaging over the two experiments, the preview benefit at Ordinal $\mathrm{Ob}$ ject Positions 1 through 4 were 119, 98, 46, and $114 \mathrm{msec}$, respectively, as reflected by mean first fixation duration, and 187, 132, 144, and $131 \mathrm{msec}$, as reflected by mean gaze duration. Counter to the cumulative preview hypothesis, the gaze duration function is relatively flat, while the first fixation duration function actually shows some tendency (significant in Experiment 1) for the amount of preview benefit to decrease rather than increase across multiple extrafoveal previews.

It might at first appear odd that the amount of preview benefit derived from an extrafoveal object would not increase with multiple previews. However, at least two models can be proposed to explain how extrafoveal information is acquired, and why there is a lack of cumulative preview benefit. Both of these models assume that covert visual attention is integral in the utilization of extrafoveal preview information to derive a preview benefit in object identification. The first model bears a strong resemblance to the "zoom lens" model of visual attention advocated by Eriksen (Eriksen \& St. James, 1986; Eriksen \& Yeh, 1985; see also LaBerge, 1983). According to this model, the first fixation on a display is associated with a wide distribution of attention over the whole display, at which time extrafoveal information is acquired from all objects simultaneously, providing some information about the objects' identities and their spatial locations (see Loftus, 1983). Thereafter, attention is narrowed down to the size of an individual object as each object is fixated, so that the detailed information required for identification can be acquired. Since attention is focused on the fixated object, no new extrafoveal information is gained after the first fixation on the display. The reduction in preview benefit over ordinal positions could be explained by such an account, if one assumes that the extrafoveal information gained during the first fixation on each display decays with time or with processing load.

The second model is based on the hypothesis that visual attention is generally directed at most toward two spatial locations, the current foveal location and the location toward which the eyes are being programmed to move next. This sequential attention hypothesis draws on work indicating that visual attention can move independently of the fovea (e.g., Posner \& Cohen, 1984; Remington, 1980; Shulman, Remington, \& McLean, 1979; Tsal, 1983). A version of this type of model, proposed by Morrison (1984; see also McConkie, 1979) to account for the use of parafoveal information in reading, states that attention begins at the point of fixation in order to allow detailed processing of the foveal stimulus, but then moves ahead to the next position once foveal processing reaches some threshold of information acquisition. The eye then follows the movement of attention to the new position once the motor programming necessary for a saccadic eye movement has been computed. Since, in this model, extrafoveal preview information is derived only from the single position to which attention has moved (see Loftus, 1972; Rayner, McConkie, \& Ehrlich, 1978; Shepherd et al., 1986, for some supporting evidence), it can account for the fact that extrafoveal preview information does not accumulate over multiple previews. Furthermore, if some resource-intensive processing must still be performed on the foveal object once attention has moved on, the amount of information from the extrafoveal object that can be used may be reduced (Henderson, 1988; Henderson \& Ferreira, 1987; Rayner, 1986). In Experiments 1 and 2, the reduction in preview benefit over ordinal position might be explained by the fact that as ordinal position increases, so does the difficulty of remembering all of the objects so far identified.

A final hypothesis regarding the lack of a cumulative preview benefit is that the subjects were taking advantage of the postview provided in the full display condition. That is, the subjects may have fixated objects in the full display condition for less time because they were able to "look back' attentionally at the object just fixated. On this hypothesis, no cumulative preview benefit is found, because attention tends to be focused on the foveal object and the object just fixated. A variant of the postview hypothesis is that attention is focused on the currently fixated object, the object fixated just prior to the currently fixated object, and the object about to be fixated next. In this case, the total "preview" benefit for an object would be the sum of the benefit derived from the preview and postview of the object. Both variants of the postview hypothesis contrast with the sequential attention model, which states that attention generally moves forward to the next position rather than backward to the last. In fact, in the strongest version of the sequential attention model, extrafoveal information can only be acquired from the next position to be fixated, because of a time-locked link between visual attention and the oculomotor system (see Morrison, 1984). The purpose of Experiment 3 was to evaluate these alternative models of the relationship between covert visual attention and extrafoveal information use.

\section{EXPERIMENT 3}

The general procedure of Experiment 3 followed that of Experiments 1 and 2. The subjects were shown displays of four objects arranged at the corners of an imaginary square, and they were asked to look at the displays while their eye movements were recorded. The subjects were instructed to begin by fixating the center of the square between the object positions; then they looked at the four objects in each display, in a prescribed order; finally they pressed a key to terminate the display. Their task was to look at each display to determine what the four objects were. After terminating the display, each subject was verbally given the name of an object by the experimenter and asked to indicate whether that object had 
appeared in the display just examined. Because of the manner in which the preview conditions used in this experiment were defined (see below), the locations from which data were examined were the second and third ordinal object positions.

In order to fully explore the acquisition of extrafoveal information for integration across saccades, five window conditions were used. Two of these, the full display and one-object conditions, were identical to those used in Experiments 1 and 2 . In the full display condition, all four objects were continuously displayed (i.e., they were potentially available for analysis) on the screen. In the one-object condition, only the object currently under fixation was displayed. Additionally, in the one + next condition, the object currently under fixation and the extrafoveal object about to be fixated in the prescribed sequence were the only objects displayed during a given fixation, while in the one + last condition, the object currently under fixation and the extrafoveal object last fixated were displayed. Finally, in the zoom condition, all four objects were displayed while the subject was fixating the initial center position in the imaginary square, but once fixation had left the center of the display, this condition was identical to the one-object condition. Figure 2 shows each window condition as a function of eye position.

In the conditions in which fewer than all four objects were displayed on a fixation (one-object, one + next, one + last, and zoom conditions), each object outside of the window was replaced by the pattern mask that was used in Experiments 1 and 2.

\section{Method}

Subjects. Ten members of the University of Massachusetts subject pool participated in Experiment 3. All of the subjects had participated in eye movement experiments previously.

Materials. Eighty line drawings of common objects drawn from the same pool as in Experiments 1 and 2 were used. The subjects again named the individually presented objects before the experiment. The stimulus displays were similar to those used in Experiments 1 and 2 . Five lists of 20 displays each were constructed such that (1) each object appeared in one display on every list, and always with 3 new objects; and (2) each object always appeared in the same location in a display across lists. The displays created in the above manner were randomly ordered on each list, and two practice trials containing objects not used in the test displays were added at the beginning of each list.

Apparatus. The apparatus was the same as that used in Experiments 1 and 2 . The program controlling the experiment was modified in order to effect the appropriate fixation-contingent display changes.

Procedure. The procedure was the same as in Experiments 1 and 2, apart from the following exceptions. After calibration of the eye tracking system, five blocks of 22 trials each were given. The first 2 trials in each block were practice and were not scored. The next 20 trials in each block constituted the test trials.

Each trial consisted of the same events as in Experiments 1 and 2. Again, after each trial, the experimenter asked the subject whether a particular object had appeared in that display. Half of the questions in each list required a "yes" response, and half a "no" response. On the "yes" trials, the subjects were queried about each of the four object positions equally often. Since each subject saw 100 test trials, and since there were 80 test objects, 20 of the objects were used as question objects twice. These objects were selected randomly, as were the objects that required a "yes" or "no" response. The subjects again had no difficulty in answering these questions correctly (see Note 3 ).

The display changes occurred in the following manner: The program controlling the experiment divided the display into five areas, four of them equivalent to the four quadrants in which an object could occur, and one a small area at the center of the display. In the one-object, one + next, and one + last conditions, a pattern mask occurred at each of the four object positions until the subject moved his/her eyes from the center. At that time, the object occupying the quadrant in which the eyes were moving (Quadrant 1) was displayed. For the one + next condition, the object in Quadrant 2 also was displayed as soon as the eyes were within Quadrant 1. When the eyes entered Quadrant 2, the object in Quadrant 2 alone was displayed in the one-object condition, the objects in Quadrants 1 and 2 were displayed in the one + last condition, and the objects in Quadrants 2 and 3 were displayed in the one + next condition. When the eyes entered Quadrant 3, the displays mirrored those for Quadrant 2. Finally, when the eyes entered Quadrant 4, the object in Quadrant 4 alone was displayed in both the one-object and one + next conditions, while the objects in Quadrants 3 and 4 were displayed in the one + last condition.

In the full and zoom conditions, all four objects were displayed until the subject moved the eyes from the center of the display to the first object position. Thereafter, all four objects continued to be displayed on each fixation regardless of eye position in the full condition. In contrast, the zoom condition was identical to the oneobject condition once the eyes had left the center of the display. Figure 2 illustrates each condition as a function of eye position. It is also important to note that the display changes occurred as a function of eye position, not as a function of the experimenterdefined order of viewing. Therefore, if a subject looked back to a particular quadrant, the display change defined for the object in that quadrant in that condition would occur. This allowed the subjects to "look back" at an object and therefore engage in more natural viewing. The display changes defined by a particular condition took place as soon as the eyes crossed a quadrant boundary (the boundaries were not visible to the subjects) and were completed in about 5 msec.

Each subject participated in five blocks of trials, each block consisting of the presentation of one display list in one preview condition. Two orderings of list presentation were used, one for each group of 5 subjects. Within a group of 5 subjects, the order of list presentation remained constant, but the preview condition was Latinsquared across those 5 subjects. This means that within a group of 5 subjects, list was confounded with practice, but that preview condition was orthogonal to both list and practice. Each subject received a short rest between blocks, and the entire experiment lasted from 45 to $60 \mathrm{~min}$.

\section{Results and Discussion}

The following analyses exclude trials on which (1) the eyetracker lost track of the eye (1\%), and (2) an object was skipped $(1 \%)$ or fixated for less than $100 \mathrm{msec}$ or more than $1000 \mathrm{msec}$, or in excess of 2 standard deviations from the mean for that condition (5\%). Table 3 presents the fixation durations for the five preview conditions at Ordinal Positions 2 and 3. Durations of fixation on objects in Quadrant 2 were marginally faster than those in Quadrant $3[F(1,9)=3.97, p<.10]$, but since position did not interact with preview condition $(F=1.4)$, this factor will not be discussed further.

We will again concentrate on the mean first fixation durations, since it is clear that the gaze duration data mirror those derived from first fixations. An analysis of vari- 
Ful1

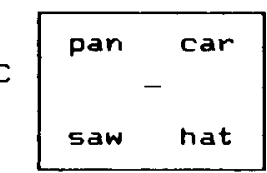

1 \begin{tabular}{ll} 
pari & car \\
saw & hat \\
\hline
\end{tabular}
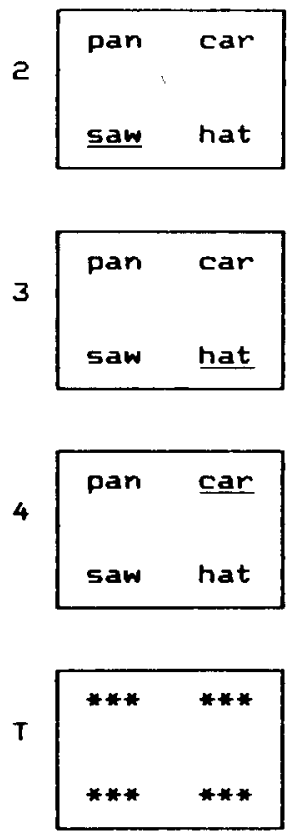

One
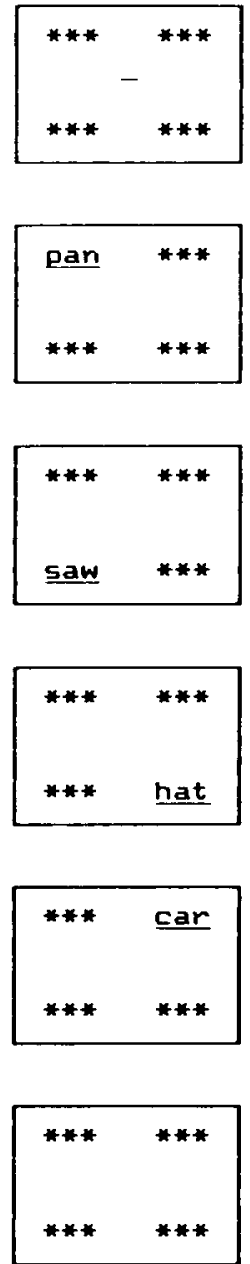

Zoom
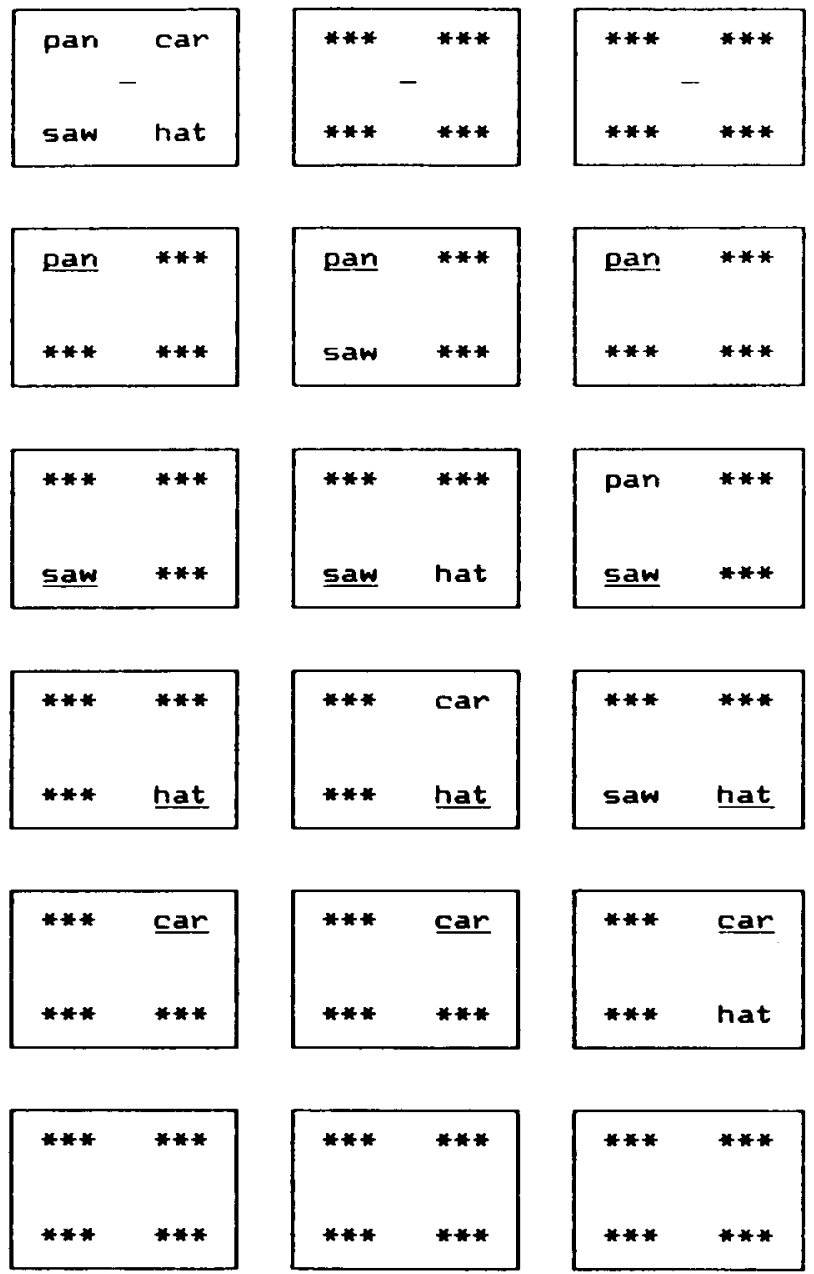

Figure 2. Schematic representation of the five window conditions used in Experiment 3, as a function of fixation position. Columns represent window conditions. Rows represent fixation positions $(C=$ center, 1 to $4=$ the quadrants, $\mathbf{T}=$ display termination). The words in each panel denote pictures, the asterisks denote the mask, and the underline shows fixation position.

ance confirmed that the effect of preview condition discernible in Table 3 was reliable $[F(4,36)=4.38$, $p<.01]$.

In order to examine the acquisition of extrafoveal information more thoroughly, several planned contrasts were conducted to distinguish among the models we discussed above. First, according to the zoom model, each trial begins with attention spread over the entire display, and preview information is acquired from all objects at that time. Thereafter, as each object is fixated, attention is devoted to the acquisition of detailed information from the fixated object and no further preview information is acquired. The fact that the zoom condition shows no preview benefit over the one-object condition $(F<1)$ rules against this model. Second, according to either postview model, some or all of the preview benefit is due to the acquisition of information from the previously fixated object during fixation of the current object. The fact that the one + last condition shows no preview benefit over the one-object condition $(F<1)$ likewise rules against any postview model.

In contrast to the attentional zoom and postview models, the sequential attention model fares quite well. According to this model, extrafoveal preview information is acquired from the object that occupies the location toward which the next eye movement will proceed. Average fixation durations when the object to be fixated next was available (full display and one + next conditions) were significantly shorter than were average fixation durations when the object to be fixated next was not available (zoom, one + last, and one-object conditions) $[F(1,9)=11.9$, $p<.01]$. In addition, considered individually, the full 
Table 3

Mean First Fixation Duration (in msec) by Preview Condition and Ordinal Object Position, Experiment 3

\begin{tabular}{cccc} 
& \multicolumn{2}{c}{ Ordinal Object } & \\
\cline { 2 - 4 } Position & 3 & Mean \\
\hline Preview Condition & 2 & 331 & 358 \\
Full display & 386 & $(488)$ & $(464)$ \\
& $(440)$ & 381 & 420 \\
One-object & 459 & $(595)$ & $(592)$ \\
& $(590)$ & 402 & 422 \\
Zoom & 444 & $(597)$ & $(617)$ \\
& $(636)$ & 353 & 376 \\
One + next & 399 & $(479)$ & $(482)$ \\
& $(485)$ & 411 & 419 \\
One +last & 429 & $(610)$ & $(580)$ \\
& $(550)$ &
\end{tabular}

Note-Mean gaze durations are given in parentheses.

display and one + next conditions both differed from the average of the zoom, one + last, and one-object conditions $[F(1,9)=11.9, p<.01$, and $F(1,9)=6.11, p<.05$, respectively].

Statistically, there was no difference in fixation times between the one + next and the full display conditions $(F<1)$. To the extent that the $18-\mathrm{msec}$ advantage of the full display condition over the one + next condition requires explanation, it may be that the display changes that occurred in all conditions apart from the full display condition produced a small increase in fixation times. More pertinent theoretically is the possibility that on some small percentage of trials, when an attentional fixation on the next object to be fixated produced full identification, attention could then have moved ahead to the object to be fixated after that, giving it a small advantage (see Morrison, 1984). Since attention could move ahead to object $n+2$ only in the full display condition, this additional benefit could only occur in the full display condition. This explanation would predict some amount of cumulative preview benefit in Experiments 1 and 2, but if the effect is on the order of $18 \mathrm{msec}$, it may have been too subtle to detect in the earlier experiments.

\section{GENERAL DISCUSSION}

The main issue addressed in this study is the relationship between covert visual attention and the availability of extrafoveal information at various locations in the visual field. In contrast to previous experimenters who have examined covert attention under static viewing conditions, we were particularly interested in the relationship between covert attention and the acquisition of extrafoveal information for integration across saccadic eye movements. By employing a contingent display change technique and allowing subjects to move their eyes as they identified objects, we were able to show that most extrafoveal information is acquired from the object to be fixated next. In Experiments 1 and 2, we found that subjects gained no more preview benefit from multiple extrafoveal glimpses of an object than from a single extrafoveal glimpse. In Experiment 3, we ruled out several alternative accounts and provided evidence that the limited preview effect results from the fact that extrafoveal preview information is primarily acquired from the object about to be fixated.

In order to account for the data presented here, we propose what we call the sequential attention hypothesis. According to this hypothesis, which draws heavily on Morrison's (1984) elaboration of McConkie's (1979) model of oculomotor control in reading, covert visual attention begins each fixation focused on the object occupying the foveal region. However, once some preset threshold of information acquisition at the fovea is reached, the attention shifts to an extrafoveal location and allows the recognition unit corresponding to the object at that location to be activated (see Pollatsek et al., 1988). If the information acquired in this way is sufficient to allow full identification before the eyes have moved, attention may shift again. However, if the extrafoveal object has not been fully identified by the time eye movement programming is completed, the object will be fixated and the activation acquired from the extrafoveal processing will speed its identification.

According to the sequential attention hypothesis, visual attention and the oculomotor system are functionally coupled. However, we do not wish to imply that movements of attention cannot occur independently of movements of the eyes. There is ample behavioral evidence for attentional movements independent of eye movements (see, e.g., Bashinski \& Bacharach, 1980; Eriksen \& Yeh, 1985; Posner, 1980; Posner \& Cohen, 1984; Remington, 1980; Shulman et al., 1979; Tsal, 1983). In fact, Posner (1980) found that subjects could move attention in the direction opposite to that in which they moved their eyes, and the patients described by Posner, Cohen, and Rafal (1982) could, with an extended latency, covertly orient their attention in a direction in which they could not make an eye movement. Our argument is that under normal circumstances, shifts of covert visual attention precede a saccadic eye movement so that movements of covert attention and the eyes are functionally, though not structurally, linked.

An issue that remains is the degree to which the sequential attention model generalizes from the experimental paradigm used in this paper to other, more ecologically valid visual tasks such as reading and scene perception. In reading, results of experiments that employ the moving window technique consistently show that readers make use of extrafoveal information up to 15 letter spaces to the right of their fixation point (McConkie \& Rayner, 1975; Rayner, 1986), while they seldom make use of information to the left of their fixation point beyond the beginning of the word that is currently being fixated (McConkie \& Rayner, 1976; Rayner, Well, \& Pollatsek, 1980). Hebrew readers, who read from right to left, show the opposite pattern, acquiring extrafoveal information predominantly from the left of their fixation point (Pollatsek, Bolozky, Well, \& Rayner, 1981). These results support the view that most extrafoveal information is acquired from the location to which the eyes are about to 
move. Also consistent with this view, Inhoff, Pollatsek, Posner, \& Rayner (in press) have found that when readers of English are forced to read from right to left, the direction from which useful extrafoveal information is acquired reverses, so that now they acquire information from the left of fixation. This last result suggests that the asymmetry (to the right in English, to the left in Hebrew) of extrafoveal information use in reading is not based upon long-term learning, but is instead malleable, as would be expected of an attentional process.

During reading, the assumption can be made that attention generally moves to the word immediately to the right of fixation (Morrison, 1984). Similarly, in the paradigm employed in this paper, the observer was given a fixation pattern to follow and thus could move attention to the next object in the prescribed sequence. During scene perception (e.g., when viewing either a picture or the actual three-dimensional world), the application of the sequential attention model is complicated by the fact that there is little constraint on potential fixation locations. Since the sequential attention model assumes that attention precedes an eye movement to a specified location, this location must be chosen prior to the attentional movement. How locations are chosen for fixation during scene perception is far from clear. One possibility (suggested by Loftus, 1983) is that during the first fixation on a scene, attention expands over the entire scene so that potential fixation locations can be chosen on the basis of semantic informativeness. Though we found no evidence for such a "zoom" mode of processing in our paradigm, it is still possible that it may operate in scene perception. A second possibility is that potential fixation positions are computed preattentively and are based upon low-level stimulus attributes such as contour density or figure-ground segregation. Unfortunately, these thoughts are at this point speculative. They underscore the need for further research in this area.

A visual attention system like that embodied in the sequential attention model can be seen to serve two general functions. First, the ability to move visual attention independently of eye movements may be in part a solution to the problem imposed by the constraint that the quality of visual information varies across the visual field. In the same way that eye movements serve to bring new areas of the visual field into the fovea and therefore increase the quality of the information derived from those areas, attentional movements may serve to increase the amount of processing resources devoted to a particular area of the visual field. Devoting attention to a particular area of the visual field may function to (1) "boost the gain" on feature extraction (Prinzmetal, Presti, \& Posner, 1986), (2) prevent cross talk between features arriving from different spatial channels (Pollatsek \& Digman, 1977); (3) allow combinatorial processes to operate over feature-based representations (Treisman \& Gelade, 1980), and/or (4) allow decision processes to serially activate internal or external responses (Duncan, 1981).

A second related function of a moving visual attention involves practicality: attention can shift location faster than the eye can move. It takes an appreciable amount of time (about $150 \mathrm{msec}$ ) for the oculomotor system to program and execute an eye movement (Rayner, Slowiaczek, Clifton, \& Bertera, 1983; Salthouse \& Ellis, 1980). On the other hand, Rayner et al. (1981) provided evidence that in reading, $50 \mathrm{msec}$ of foveal input provides enough visual information to allow word identification. It seems, therefore, that there are well over $100 \mathrm{msec}$ of lag time during a fixation-from the point in time when the foveal stimulus has been processed sufficiently to allow identification, until the eye can move on to the next stimulus. The sequential attention model suggests that rather than wait the additional time for an eye movement to be completed, the visual system begins to process a new location in the visual field by moving visual attention. The eye movement then follows this attentional movement as quickly as it can-that is, once the motor programming necessary for the eye movement has been completed.

The sequential attention model is consistent with extant neuropsychological data on the relationship between visual attention and the oculomotor system. For example, it has been found that the discharge of cells in the superior colliculus and the frontal eye fields of the alert monkey are selectively enhanced prior to an eye movement when their receptive fields are in the area to which the eyes are being programmed to move (Goldberg \& Bushnell, 1982; Goldberg \& Wurtz, 1972). Since these effects are contingent on the occurrence of an eye movement, they may reflect strictly oculomotor (rather than attentional) processes (Wurtz, 1986). On the other hand, the fact that an eye movement is necessary to produce selective enhancement in these areas does not rule out an attentional component. Less ambiguously, neurons in the parietal cortex (Area 7) show enhanced discharge when a stimulus falling in its receptive field is actively used, including but not limited to when it is used as the target for a saccadic eye movement (Bushnell, Goldberg, \& Robinson, 1981). Robinson and Peterson (1986) interpreted the results on parietal neurons as indicating a "shift of visual attention common to both the saccade and peripheral attention tasks"' (p. 151). Finally, Posner et al. (1982) found that human patients suffering from midbrain damage showed vastly slowed covert orienting in the direction of impaired eye movements. This result again suggests a functional link between attentional movements and the oculomotor system.

In summary, we suggest that in complex visual tasks such as object identification, covert attentional movements ordinarily precede the eyes toward the location which is to be fixated next, and that extrafoveal visual information is primarily acquired from that location at the exclusion of other locations.

\section{REFERENCES}

Balota, D. A., Pollatsek, A., \& Rayner, K. (1985). The interaction of contextual constraint and parafoveal visual information in reading. Cognitive Psychology, 17, 364-390.

BASHinski, H. S., \& BACHARACh, V. R. (1980). Enhancement of per- 
ceptual sensitivity as the result of selectively attending to spatial locations. Perception \& Psychophysics, 28, 241-248.

Bushnell, M. C., Goldberg, M. E., \& Robinson, D. L. (1981). Behavioral enhancement of visual responses in monkey cerebral cortex: I. Modulation in posterior parietal cortex related to selective visual attention. Journal of Neuropsychology, 46, 755-772.

Duncan, J. (1981). Directing attention in the visual field. Perception \& Psychophysics, 30, 90-93.

Eriksen, C. W., \& ST. JAmes, J. D. (1986). Visual attention within and around the field of focal attention: A zoom lens model. Perception \& Psychophysics, 40, 225-240.

ERIKSEN, C. W., \& YEH, Y. (1985). Allocation of attention in the visual field. Journal of Experimental Psychology: Human Perception \& Performance, 11, 583-597.

Friedman, A. (1979). Framing pictures: The role of knowledge in automatized encoding and memory for gist. Joumal of Experimental Psychology: General, 108, 316-355.

Goldberg, M. E., \& BUShNell, M. C. (1982). Behavioral enhancement of visual responses in monkey cerebral cortex: II. Modulation in frontal eye fields specifically related to saccades. Joumal of Neurophysiology, 46, 773-787.

GoldBerG, M. E., \& WURTZ, R. H. (1972). Activity of superior colliculus in behaving monkey: II. Effect of attention on neuronal responses. Journal of Neurophysiology, 35, 560-574.

HENDERSON, J. M. (1988). Visual attention and the acquisition of extrafoveal information during eye fuxations. Unpublished doctoral dissertation, University of Massachusetts, Amherst, Massachusetts.

Henderson, J. M., \& Ferreira, F. (1987, September). Visual attention and the perceptual span in reading. Paper presented at the Annual Joseph R. Royce Research Conference, University of Alberta, Edmonton, Alberta.

Henderson, J. M., Pollatsek, A., \& Rayner, K. (1987). Effects of foveal priming and extrafoveal preview on object identification. Joumal of Experimental Psychology: Human Perception \& Performance, 13, 449-463.

HutTenlocher, J., \& KUBiceK, L. F. (1983). The source of relatedness effects on naming latency. Journal of Experimental Psychology: Learning, Memory, \& Cognition, 9, 486-496.

INHOFF, A. W. (1984). Two stages of word processing during eye fixations in the reading of prose. Journal of Verbal Learning \& Verbal Behavior, 23, 612-624.

Inhoff, A. W., Pollatsek, A., Posner, M. I., \& Rayner, K. (in press). Covert attention and eye movements during reading. Quarterly Journal of Experimental Psychology.

KLEIN, R. (1980). Does oculomotor readiness mediate cognitive control of visual attention? In R. S. Nickerson (Ed.), Attention and Performance VIII (pp. 259-276). Hillsdale, NJ: Erlbaum.

Kroll, J. F., \& Potter, M. C. (1984). Recognizing words, pictures, and concepts: A comparison of lexical, object, and reality decisions. Journal of Verbal Learning \& Verbal Behavior, 23, 39-66.

LABERGE, D. (1983). Spatial extent of attention to letters and words. Journal of Experimental Psychology: Human Perception \& Performance, 9, 371-379.

Lim A, S. D. (1987). Morphological analysis in sentence reading. Journal of Memory \& Language, 26, 84-99.

LofTUS, G. R. (1972). Eye fixations and recognition memory for pictures. Cognitive Psychology, 3, 525-551.

LofTUs, G. R. (1983). Eye fixations on text and scenes. In K. Rayner (Ed.), Eye movements in reading. New York: Academic Press.

LofTus, G. R., \& MACKWORTH, N. H. (1978). Cognitive determinants of fixation location during picture viewing. Journal of Experimental Psychology: Human Perception \& Performance, 4, 565-572.

McConkie, G. W. (1979). On the role and control of eye movements in reading. In P. A. Kolers, M. E. Wrolstad, and H. Bouma (Eds.), Processing of visible language (pp. 37-48). New York: Plenum Press.

McConkie, G. W., \& Rayner, K. (1975). The span of the effective stimulus during a fixation in reading. Perception \& Psychophysics, 17, 578-586.

MCConkie, G. W., \& Rayner, K. (1976). Asymmetry of the perceptual span in reading. Bulletin of the Psychonomic Society, 8, 365-368.
McConkiE, G. W., \& ZoLA, D. (1979). Is visual information integrated across successive fixations in reading? Perception \& Psychophysics, 25, 221-224

MoRRIS, R. (1987). Eye movement guidance in reading: The role of parafoveal letter and space information. Unpublished master's thesis, University of Massachusetts, Amherst, Massachusetts.

Morruson, R. E. (1984). Manipulation of stimulus onset delay in reading: Evidence for parallel programming of saccades. Journal of $E x-$ perimental Psychology: Human Perception \& Performance, 10 , 667-682.

Pollatsek, A., Bolozky, S., Well, A. D., \& Rayner, K. (1981) Asymmetries in the perceptual span for Israeli readers. Brain \& Language, 14, 174-180.

Pollatsek, A., \& Digman, L. (1977). Dependent spatial channels in visual processing. Cognitive Psychology, 9, 326-352.

Pollatsek, A., Rayner, K., \& Collins, W. E. (1984). Integrating pictorial information across eye movements. Journal of Experimental Psychology: General, 113, 426-442.

Pollatsek, A., Rayner, K., \& Henderson, J. M. (1988). The role of spatial location in the integration of pictorial information across saccades. Manuscript submitted for publication.

POSNER, M. I. (1980). Orienting of attention. Quarterly Journal of Experimental Psychology, 32A, 3-25.

Posner, M. I., \& CohEN, Y. (1984). Components of visual orienting. In H. Bouma \& D. Bowhuis (Eds.), Attention and Performance $X$ (pp. 531-556). Hillsdale, NJ: Erlbaum.

Posner, M. I., Cohen, Y., \& Rafal, R. D. (1982). Neural systems control of spatial orienting. Philosophical Transactions of the Royal Society of London, B298, 187-198.

Prinzmetal, W., Presti, D. E., \& Posner, M. I. (1986). Does attention affect visual feature integration? Journal of Experimental Psychology: Human Perception \& Performance, 12, 361-369.

RAYNER, K. (1986). Eye movements and the perceptual span in beginning and skilled readers. Joumal of Experimental Child Psychology, 41, 211-236.

RAYNER, K., \& BERTERA, J. H. (1979). Reading without a fovea. Science, 206, 468-469.

RAyner, K., Inhoff, A. W., Morrison, R. E., Slowiaczek, M. L. \& Bertera, J. H. (1981). Masking of foveal and parafoveal vision during eye fixations in reading. Journal of Experimental Psychology: Human Perception \& Performance, 7, 167-179.

Rayner, K., McConkie, G. W., \& Ehrlich, S. (1978). Eye movements and integrating information across fixations. Journal of $E x-$ perimental Psychology: Human Perception \& Performance, 4, 529-544.

Rayner, K., \& Pollatsek, A. (1987). Eye movements in reading: A tutorial review. In M. Coltheart (Ed.), Attention and Performance $X I I$ (pp. 327-362). London: Erlbaum.

Rayner, K., Slowiaczek, M. L., Clifton, C., \& Bertera, J. H (1983). Latency of sequential eye movements: Implications for reading. Journal of Experimental Psychology: Human Perception \& Performance, 9, 912-922.

Rayner, K., Well, A. D., \& Pollatsek, A. (1980). Asymmetry of the effective visual field in reading. Perception \& Psychophysics, 27, 537-544.

Rayner, K., Well, A. D., Pollatsek, A., \& Bertera, J. H. (1982). The availability of useful information to the right of fixation in reading. Perception \& Psychophysics, 31, 537-550.

Remington, R. W. (1980). Attention and saccadic eye movements. Journal of Experimental Psychology: Human Perception \& Performance, 6, 726-744.

Robinson, D. L., \& Peterson, S. E. (1986). The neurobiology of attention. In J. E. LeDoux and W. Hirst (Eds.), Mind and brain: Dialogues in cognitive neuroscience (pp. 142-171). New York: Cambridge University Press.

Salthouse, T. A., \& Eluis, C. L. (1980). Determinants of eye-fixation duration. American Journal of Psychology, 93, 207-234.

ShePherd, M., Findlay, J. M., \& Hockey, R. J. (1986). The relationship between eye movements and spatial attention. Quarterly Journal of Experimental Psychology, 38A, 475-491. 
Shulman, G. L., Remington, R. W., \& McLean, J. P. (1979). Moving attention through visual space. Journal of Experimental Psychology: Human Perception \& Performance, 5, 522-526.

SNodgrass, J. G., \& Vanderwart, M. (1980). A standardized set of 260 pictures: Norms for name agreement, image agreement, familiarity, and visual complexity. Journal of Experimental Psychology: $\mathrm{Hu}$ man Learning \& Memory, 6, 174-215.

Treisman, A., \& Gelade, G. (1980). A feature-integration theory of attention. Cognitive Psychology, 12, 97-136.

TSAL, Y. (1983). Movements of attention across the visual field. Journal of Experimental Psychology: Human Perception \& Performance, 9. 523-530.

Wurtz, R. H. (1986). Stimulus selection and conditional response mechanisms in the basal ganglia of the monkey. In M. I. Posner and O. S. M. Marin (Eds.), Attention and Performance XI (pp. 44I-455). Hillsdale, NJ: Erlbaum.

\section{NOTES}

1. Twelve filler displays were also given in each block. As these were part of a separate study, they will not be discussed further, and are relevant here only to the extent that they helped prevent memorization of the test displays across blocks.

2. In previous work, we found that when subjects were allowed to look at objects in displays similar to these in any order that they chose, they tended to spontaneousiy adopt a stereotypical viewing pattern across displays; see Henderson et al., 1987.

3. Errors did not exceed $1 \%$ for any subject in any of the three experiments, and most subjects made no errors.

(Manuscript received April 10, 1987; revision accepted for publication August 29, 1988.) 